\title{
Pulmonary infiltrates and adult acute leukaemia: empirical treatment and survival related to the extent of pulmonary radiological disease
}

\author{
AG WARDMAN, DW MILLIGAN, JA CHILD, IW DELAMORE, NJ COOKE \\ From the Departments of Respiratory Medicine and Haematology, General Infirmary, Leeds, and \\ Department of Clinical Haematology, Manchester Royal Infirmary, Manchester
}

\begin{abstract}
In a retrospective review of 141 consecutive adult patients with acute leukaemia there were 33 local and eight diffuse parenchymal pulmonary abnormalities noted on the first abnormal chest radiograph. Empirical treatment was given without any invasive diagnostic procedures. Twenty of the 41 patients $(49 \%)$ with radiographic abnormalities died-10 of 31 patients $(32 \%)$ in the "local" group (excluding two who were not actively treated) and all eight patients in the "diffuse" group. One patient in the local group and six in the diffuse group died within 48 hours of the first abnormal chest radiograph. The results in the local group suggest that failure to respond to the initial treatment prescribed would allow time to perform an invasive procedure in most patients that may lead to a change in management. In the diffuse group, however, an early invasive procedure would be required if the result were to have a chance to influence the outcome.
\end{abstract}

Infection remains the major cause of death in adult acute leukaemia and the lung is the commonest organ affected. ${ }^{\prime 2}$ Septicaemia frequently complicates pneumonia, though the gastrointestinal tract may be the more important source of infection. ${ }^{3}$ Gram negative bacteria are the most frequent cause of pneumonia, but Gram positive and anaerobic bacteria, Pneumocystis carinii, fungi, and other opportunistic pathogens are important. Pulmonary oedema, haemorrhage, and leukaemic infiltration occur and more than one of these pathological processes and infections may be present. ${ }^{14}$ The reported mortality associated with pulmonary infiltration remains high at $60 \% .^{5}$

Postmortem series from the United States ${ }^{146}$ showed that in many cases the underlying cause of pulmonary infiltration, particularly opportunistic infections and leukaemic infiltrates, was not diagnosed in vivo and this led to the enthusiastic application and assessment of various lung biopsy techniques. $^{5-17}$ Though doubt has been expressed on the

Address for reprint requests: Dr NJ Cooke, Department of Respiratory Medicine, General Infirmary, Leeds LS1 3EX.

Accepted 21 May 1984 relevance of American to British $^{3}$ findings we are not aware of any British postmortem series for comparison. With invasive procedures a specific diagnosis can be made in up to $60-70 \%$ of patients, compared with $20-30 \%$ when only conventional investigations are carried out. The alternative approach to treatment is based on conventional investigation and on clinical and radiological evidence, and uses the collective knowledge obtained from postmortem and biopsy series; this empirical approach to management remains popular in the United Kingdom and requires the administration of broad spectrum antibiotics, high dose cotrimoxazole, platelet transfusion if the count is less than $10 \times 10^{\%} / 1$, and administration of diuretics if pulmonary oedema is suspected. Fungal infections may be the cause of $12-25 \%^{718}$ of infiltrates and delay in initiating treatment with amphotericin B may be disadvantageous.

There is no report of the outcome of the purely empirical approach to treatment in a consecutive unselective series of adults with acute leukaemia and pulmonary infiltrates in the United Kingdom. Such a survey may stimulate discussion of the place and timing of invasive procedures in this complex clinical condition. 


\section{Patients and methods}

The case records and chest radiographs of all newly diagnosed patients aged over 14 years with acute myeloid (AML) and acute lymphoblastic (ALL) leukaemia from two regional centres, Manchester Royal Infirmary and Leeds General Infirmary, were reviewed; Manchester records were from January 1979 to December 1981 and the Leeds records from January 1978 to December 1981 . There were 141 patients, 48 from Leeds General Infirmary and 93 from Manchester Royal Infirmary. One hundred and eight patients had AML and 33 ALL. The mean age at diagnosis was 49 years for AML and 31 for ALL. There was no significant difference in age, sex, or median probability of survival between the two centres for either AML or ALL, suggesting that the two hospital groups were homogeneous. All patients received standard induction remission treatment as part of the appropriate AML or ALL Medical Research Council trial. For patients with AML the principal drugs used in induction were doxorubicin, cytosine, arabinoside, and 6-thioguanine; and for ALL prednisolone and vincristine. Most patients did not receive antibiotic prophylaxis during induction, though nystatin, amphotericin lozenges, or miconazole gel were used as part of routine mouth care. Patients were examined daily and in febrile patients repeat urine, nasal, throat perineal, and blood cultures were taken. A temperature of $38^{\circ} \mathrm{C}$ or more was an indication for broad spectrum antibiotic treatment. Chest radiographs were taken in patients with unexplained fever or respiratory symptoms and were repeated as clinically indicated.

Chest radiographic parenchymal abnormalities were reviewed by two of the authors and classified as "local" or "diffuse." Local changes were defined as being of lobar or segmental distribution affecting one or more lobes but not all zones, whereas diffuse abnormalities required changes to be present in all zones. The presence of mediastinal lymphadenopathy and pleural effusion was also recorded. Where a radiographic abnormality occur-

Table 1 Findings in 41 patients with chest radiographic abnormality accompanying acute leukaemia

\begin{tabular}{lccc}
\hline & $A M L$ & $A L L$ & Total \\
\hline Number of abnormalities & 34 & 7 & 41 \\
Number of "local" abnormalities & 27 & 6 & 33 \\
(number fatal) & $(10)$ & $(2)$ & $(12)$ \\
Number of "diffuse" abnormalities & 7 & 1 & 8 \\
(number fatal) & $(7)$ & $(1)$ & $(8)$ \\
\hline
\end{tabular}

$A M L$-acute myeloid leukaemia; $A L L$-acute lymphatic leukaemia. red the following contemporary clinical details were abstracted from the case records: 1-haematological state at the time, including the neutrophil count and evidence of remission or relapse; 2-the results of bacteriological and virological investigations; 3treatment given during the episode; 4-the outcome of the episode, including evidence of radiological resolution or postmortem information where available.

\section{Results}

Forty six chest radiographic abnormalities were noted from 46 of the 141 patients (33\%), of which five were not parenchymal. In the 41 remaining cases the type and nature of the radiographic abnormalities did not differ between the two centres or between the two categories of leukaemia. Table 1 shows the overall results of the two different radiographic groups with numbers of deaths in each group. In 27 of the 41 cases the patients had an associated profound granulocytopenia (neutrophil count $\left.<0.05 \times 10^{9} / \mathrm{l}\right)$ and most of these were severely thrombocytopenic. Twenty seven of the radiographic changes occurred during induction, 13 during leukaemic relapse, and only one in remission; no abnormalities were present before induction treatment was started. At the time of development of these abnormalities bacteriological and mycological cultures of blood, urine, and sputum were initiated. Throat, nasal, and perianal swabs were obtained and serum was taken for virological study. In 33 cases $(80 \%)$ no organism was isolated. Sputum culture was positive in five cases, six organisms being isolated (Escherichia coli 2, Staphylococcus aureus 2, Pseudomonas aeruginosa 1, Klebsiella sp 1). Blood culture was positive in three further cases (Escherichia coli, Staphylococcus aureus, and Klebsiella $\mathrm{sp}$ ). Granulocyte transfusions were given only when proved bacterial infection failed to respond to antibiotics.

Table 2 shows the outcome of the clinical and empirical approach adopted in the 33 patients with "local" pulmonary radiographic abnormalities. Of the 26 patients treated with antibiotics with or without amphotericin B, 10 died (38\%). Of these 10 patients, three had bilateral extensive radiographic abnormalities not affecting all lung zones. One of these three patients had never achieved a marrow remission of the acute leukaemia, and permission for necropsy was refused in the other two. Postmortem information was available for the other seven patients. In three there was extensive pulmonary haemorrhage accompanied by pulmonary oedema in one patient. The findings in the remaining four patients were pulmonary oedema alone in one, kleb- 
Table 2 Treatment and outcome in the group with local pulmonary infiltrates on radiographs

\begin{tabular}{lcc}
\hline Treatment & No & $\begin{array}{c}\text { No of } \\
\text { deaths }\end{array}$ \\
\hline Aminoglycoside plus carbenicillin or ticarcillin & 23 & 9 \\
Aminoglycoside plus carbenicillin or ticarcillin & 3 & 1 \\
$\quad$ and amphotericin B & 2 & 0 \\
Observation plus continued chemotherapy & 2 & 0 \\
Observation for probable pulmonary infarction & 1 & 0 \\
Diuretic alone & 2 & 2 \\
All active treatment withheld on grounds of & 33 & 12 \\
$\quad$ refractory leukaemia & & \\
Total &
\end{tabular}

Table 3 Interval between chest radiographic abnormality and death in 18 patients with local and diffuse radiographic abnormalities dying of acute leukaemia

\begin{tabular}{llll}
\hline Day of death & Local & Diffuse & Total \\
\hline $0-1$ & 0 & 4 & 4 \\
$1-2$ & 1 & 2 & 3 \\
$3-5$ & 2 & 1 & 3 \\
$6-11$ & 3 & 1 & 4 \\
$12-28$ & 4 & 0 & 4 \\
Total & $10 \dagger$ & 8 & 18 \\
\hline
\end{tabular}

${ }^{*}$ Refers to the number of days after initial abnormal chest radiograph.

†Excludes two patients, dying on days 5 and 19, from whom all active treatment was withheld on grounds of "refractory leukaemia."

siella pneumonia in one, and unidentified pneumonia in two.

Seven of the eight patients with "diffuse" shadowing received the same antibiotic combination as patients in the "local" group; one patient was treated with diuretics alone. All eight patients died during the episode, six within 48 hours of the initial abnormal chest radiograph. Nine of the 10 patients dying with local abnormalities did so more than 48 hours after the first identification of the radiographic changes.

Table 3 shows the details of the day of death related to the first identification of radiographic abnormality. The overall mortality associated with the radiographic abnormality in this unselected consecutive series of 41 patients was $49 \%$. The median probability of survival of the 141 patients was 397 and 697 days respectively for AML and ALL: these figures are similar to the national survival figures, suggesting that the group was representative of British practice. ${ }^{19} 20$

\section{Discussion}

The incidence of pulmonary complications in the present series is similar to that in previous studies. ${ }^{4} 18$ The difficulty of making a diagnosis by conventional non-invasive means and the association with the low neutrophil counts found at the time of remission induction and relapse are confirmed.

Comparison of the mortality rate of $49 \%$ in the present series with the rates found in other studies of the outcome of pulmonary infiltrates and pneumonia in leukaemia is difficult. Sickles $e^{a l^{7}}$ in 1973 reported a mortality of $65 \%$, Singer et al $^{5}$ in $197960 \%$, and Tenholder and Hooper ${ }^{8}$ in 1980 $45 \%$. All three series were selected and that of Tenholder and Hooper ${ }^{8}$ contained many patients without acute leukaemia. The present data suggest that selection of patients, which may have been due to the referral pattern in these series from the United States, is likely to have a major influence on results. Delay in referral will result in many patients with "diffuse" changes dying in the first 48 hours after an abnormal chest radiograph, while prompt initial treatment and a favourable response by the patient may obviate the need for specialist referral.

In patients with "local" radiographic changes $71 \%$ have been considered to have bacterial pneumonias. ${ }^{8}$ The response rate in the present series of $62 \%$ (16 of 26) in patients with "local" changes to antibiotics is in agreement with this.

The overall results in the patients with local changes in the present study, excluding the two patients in whom all treatment was withheld on account of their refractory leukaemias, give a mortality rate of $32 \%$ (10 of 31$)$. The postmortem results in the "local" group of this series suggest that at least some of the causes of death were treatable; for example, pulmonary haemorrhage had not been thought to be a major problem in life in three patients. Fibreoptic bronchoscopy with bronchoalveolar lavage ${ }^{21}$ performed at this time may have suggested the diagnosis. Unfortunately there is no published evidence that survival is improved by making a specific diagnosis, despite appropriate therapeutic changes. ${ }^{101122}$ The figures in table 3 suggest that most patients with local changes survive for at least two days after an abnormal chest radiograph.

All eight patients with diffuse radiological changes in this series died, six within the first 48 hours after the initial abnormal radiograph. Possibly patients with diffuse changes are merely at a more advanced stage of a pathological process than those presenting with local changes and if this is so it may account for the high mortality rate. There was some evidence in this series of progressive radiological changes in the local group, but no patient changed from the "local" to the "diffuse" group. Tenholder and Hooper, however, reported that in patients with diffuse radiological changes only $35 \%$ of episodes were infectious and that $93 \%$ of these were due to opportunistic infections. 
Unfortunately only one necropsy was performed in the diffuse group in this series and there is no other British necropsy information in patients with diffuse radiological changes available. The observation of the speed of death in the diffuse group in our series suggests that if the results of an invasive procedure are to have a chance of influencing clinical progress the procedure would have to be performed very soon after the finding of diffuse radiological changes. Whether an invasive procedure is to be performed or not, empirical treatment should be started without delay and should include broad spectrum antibiotic combinations, high dose cotrimoxazole, amphotericin B, diuretics, and platelet transfusion.

We gratefully acknowledge the assistance provided by the Council chartists, Mrs J Farish (Leeds General Infirmary) and Mrs C O'Rourke (Manchester Royal Infirmary), and Mrs Kathryn Marsden and Mrs Ruth Evans for secretarial assistance. The survival data were kindly provided by Mrs J Thoroughgood, medical statistician to the Yorkshire Regional Cancer Organisation.

\section{References}

${ }^{1}$ Bodey GP, Powell RD, Hersh EM, Yetevian A, Freireich EJ. Pulmonary complications of acute leukaemia. Cancer 1966;19:781-93.

${ }^{2}$ Slevin M, Rohatiner A, Malpas JS, Lister A. Pneumonia during treatment of acute leukaemia [letter]. Br Med J 1981;282: 473.

${ }^{3}$ Hayhoe FGJ, Rees JKH. Pneumonia during treatment of acute leukaemia [letter]. $\mathrm{Br} \mathrm{Med} J$ 1980;281: 1566-7.

${ }^{4}$ Chang H-Y, Rodriguez V, Narboni G, Bodey GP, Luna MA, Freireich EJ. Causes of death in adults with acute leukemia. Medicine (Baltimore) 1976;55:259-68.

${ }^{5}$ Singer C, Armstrong D, Rosen PP, Walzer PD, Yu B. Diffuse pulmonary infiltrates in immunosuppressed patients. Am J Med 1979;66:110-9.

${ }^{6}$ Mirsky HS, Cuttner J. Fungal infection in acute leukemia. Cancer 1972;30:348-52.

7 Sickles EA, Young VM, Greene WH, Wiernick PH. Pneumonia in acute leukemia. Ann Intern Med 1973;79:528-34.
${ }^{8}$ Tenholder MF, Hooper RG. Pulmonary infiltrates in leukaemia. Chest 1980;78:468-73.

${ }^{9}$ Cunningham JH, Zavala DC, Corry RJ, Keim LW. Trephine air drill, bronchial brush, and fibreoptic transbronchial lung biopsies in immunosuppressed patients. Am Rev Respir Dis 1977;115:213-20.

${ }^{10}$ Pennington JE, Feldman NT. Pulmonary infiltrates and fever in patients with haematologic malignancy. Assessment of transbronchial biopsy. Am J Med 1977;62:581-7.

1 Rossiter SJ, Craig Miller D, Chung AM, Carrington CB, Mark JBD. Open lung biopsy in the immunosuppressed patient-is it really beneficial? J Thorac Cardiovasc Surg 1979;77:338-45.

12 Lauver GL, Hasan FM, Morgan RB, Campbell SC. The usefulness of fibreoptic bronchoscopy in evaluating new pulmonary lesions in the compromised host. $A m \mathbf{J}$ Med 1979;66:580-5.

${ }^{13}$ Feldman NT, Pennington JE, Ehrie MG. Transbronchial lung biopsy in the compromised host. JAMA 1977;238:1377-9.

14 Toledo-Pereyra LH, DeMeester TR, Kinealey A, MacMahon H, Churg A, Colomb H. The benefits of open lung biopsy in patients with previous non-diagnostic transbronchial lung biopsy. A guide to appropriate therapy. Chest 1980;77:647-50.

${ }^{15}$ Leight GS, Michaelis SLL. Open lung biopsy for the diagnosis of acute diffuse pulmonary infiltrates in the immunosuppressed patient. Chest 1978;73:477-82.

${ }^{16}$ Matthay RA, Farmer WC, Odero D. Diagnostic fibreoptic bronchoscopy in the immuno-compromised host with pulmonary infiltrates. Thorax $1977 ; 32: 539-45$.

${ }^{17}$ Greenman RL, Goodall PT, King D. Lung biopsy in immunocompromised hosts. Am J Med 1975;59:488-96.

${ }^{18}$ Bodey GP, Rodriguez V, Chang H-Y, Narboni G. Fever and infection in leukemic patients. Cancer 1978;41:1610-22.

${ }^{19}$ Peto R, Pike MC, Armitage P, et al. Design and analysis of randomized clinical trials requiring prolonged observation of each patient: Analysis and examples. Br J Cancer 1977;35:1-39.

${ }^{20}$ British Medical Research Council: Rees JKH, Swirsky DM, Hayhoe FGJ. Treatment of acute myeloid leukaemia: results of a large collaborative study. In: Proceedings of the 7th Congress of the International Society of Haematology. Barcelona: International Society of Haematology, 1983:81 (abstract).

${ }^{21}$ Drew WL, Finley TN, Golde DW. Diagnostic lavage and occult pulmonary haemorrhage in thrombocytopenic immunocompromised patients. Am Rev Respir Dis 1977;116:215-21.

${ }^{22}$ Puksa S, Hutcheon MA, Hyland RH. Usefulness of transbronchial biopsy in immunosuppressed patients with pulmonary infiltrates. Thorax $1983 ; 38: 146-50$. 